\title{
Application of the Schwarz-Christoffel map to the Laplacian growth of needles and fingers
}

\author{
N. R. McDonald ${ }^{*} *$ \\ Department of Mathematics, University College London, London WC1E 6BT, United Kingdom
}

(Received 18 September 2019; published 3 January 2020)

\begin{abstract}
A numerical procedure based on the Schwarz-Christoffel map suitable for the study of the Laplacian growth of thin two-dimensional protrusions is presented. The protrusions take the form of either straight needles or curved fingers satisfying Loewner's equation, and are represented by slits in the complex plane. Particular use is made of Driscoll's numerical procedure, the SC Toolbox, for computing the Schwarz-Christoffel map from a half plane to a slit half plane. Since the Schwarz-Christoffel map applies only to polygonal regions, the growth of curved fingers is approximated by an increasing number of short straight line segments. The growth rate is given by a fixed power $\eta$ of the harmonic measure at the finger or needle tips and so includes the possibility of "screening" as the needles of fingers interact with themselves and with boundaries. The method is illustrated with examples of multiple needle and finger growth in half-plane and channel geometries. The effect of $\eta$ on the trajectories of asymmetric bifurcating fingers is also studied.
\end{abstract}

DOI: 10.1103/PhysRevE.101.013101

\section{INTRODUCTION}

Systems having an interface separating two different phases or media evolving in response to diffusion arise in many different scenarios and across widely varying scales. When diffusion is governed by Laplace's equation and the velocity of the interface is given by the gradient of the phase, the motion is known as Laplacian growth. Even in two dimensions, Laplacian growth leads to complicated, often beautiful, patterns. Examples include Saffman-Taylor fingering [1], diffusion-limited aggregation [2], the formation of ramified river valley networks by groundwater flow [3], erosion of sediment to form narrow channels in river deltas [4], combustion fronts [5], magnetic flux dendrite formation in superconductors [6], and growth of bacterial colonies [7]. In these examples instability at the interface leads to long narrow protrusions-fingers - of one phase penetrating the other.

Theoretical study of two-dimensional Laplacian growth and its resultant pattern formation involves consideration of difficult nonlinear free boundary problems. One assumption which enables progress is to assume that the fingers are infinitesimally thin and advance at their tips only with velocity proportional to the local gradient of the phase. In terms of complex analysis the fingers can be thought of as evolving slits in the complex plane. This realization coupled with conformal-mapping methods has resulted in considerable understanding of the Laplacian growth of thin fingers, e.g., $[8,9]$. A striking example of this is an explanation of the remarkable observation that in stream networks sculpted by seepage erosion the angle at which streams bifurcate is close to $2 \pi / 5$, e.g., $[3,10,11]$.

At its simplest, the thin protrusions can be modelled as straight slits in the complex plane which grow at their tips only. It is natural to map this slit domain to some canonical

\footnotetext{
*n.r.mcdonald@ucl.ac.uk
}

domain such as the upper half plane or the exterior to the unit disk. Since needles are straight the slit domain is polygonal and the mapping takes the form of a Schwarz-Christoffel map. Such needle models have been proposed as representative of growing "arms" in the star shaped clusters typically formed in diffusion-limited aggregation, e.g., [12-14]. Similar radial needlelike structures were reported in the recent numerical solution of a Laplacian growth model for evolving river deltas [4]. Theoretical results for these models are usually confined to two arms or symmetric arrangements of multiple arms. In the two arm case symmetry breaking occurs with one of the arms eventually growing at the expense of the other $[13,14]$. This effect is referred to as screening. The needle model has also been studied in a half plane in the case of a periodic "forest" of needles growing upward from the real axis. In such an arrangement with adjacent needles being of alternate lengths the screening effect can be shown to be exponential [15], i.e., the growth speed of the shorter needles becomes exponentially small as the difference in needle length grows. A complementary approach models the growth of a periodic set of parallel growing needles using a Fokker-Planck equation [16].

The growth of curved infinitesimally thin fingers can also be thought of as evolving curved slits in the complex plane. The natural mathematical approach to describe growing slits in the complex plane is to use Loewner's differential equation governing the time evolution of a conformal map from, say, a now curved slit half plane to a half plane minus the slit. The equation effectively encodes the shape of a growing slit according to some given real driving function (see Sec. II A). More generally, Loewner's equation has proved an invaluable tool in addressing questions in complex analysis since its introduction in 1923. Recently it has received considerable attention for the case when the driving function is stochastic owing to its use in understanding two-dimensional lattice models in statistical mechanics. See, for example, [17] for a historical perspective and [18] for an extensive review with 
emphasis on applications. In the Laplacian growth problem while the driving function is deterministic it needs to be found as part of the solution $[8,9,11]$.

Exact solutions of the Loewner equation in the half plane are limited to cases involving either one slit and special choices of the driving function, or symmetric arrangements of two or three slits—-see, e.g., [19-22]. Alternatively, for a given driving function, Loewner's equation can be solved numerically either by direct discretization [19], or by successively approximating the driving function over short time intervals by a function for which the Loewner equation may be solved explicitly [23].

In Laplacian growth, when the finger grows along local flow lines of the Laplacian field it is often referred to as "geodesic growth" [8]. Recently, Devauchelle et al. [11] have established the equivalence between geodesic growth, growth which maintains local symmetry in the phase field lines about the tip, and growth that maximizes flux into a finger's tip. This suggests an alternative numerical approach in which the Laplacian field is computed (e.g., using finite elements [24]) and its behavior near a given tip used to grow the finger in a direction determined by local symmetry. A similar approach is taken here: a curved finger is approximated by a series of discrete straight-line segments and can therefore be regarded as a polygon. The polygon is then mapped by the Schwarz-Christoffel transform to the upper half plane where the solution to Laplace's equation is used to find the direction along which the finger grows in order to preserve local symmetry. This paper makes extensive use of the SC Toolbox [25] to compute numerically the Schwarz-Christoffel transform.

Section II details the Laplacian growth problems considered here, namely the evolution of both straight needles and curved fingers and the rules governing their growth. A common theme to both analytical and numerical approaches of this paper is the use of the Schwarz-Christoffel transform. Its application to needle growth in the half plane is presented in Sec. III. The transform is used to derive asymptotic growth rules for a pair of needles for small and large times, and to investigate instability of pairs of needles, revealing strong screening in which one needle grows at the expense of the other. Configurations of needle pairs which are inclined to the base of the half plane are studied numerically, as well as multiple needles in both half-plane and semistrip geometries. Section IV presents a numerical procedure for computing curved finger growth and demonstrates its use by computing results for symmetric two and three finger growth. Relevant to the bifurcation of stream networks the method is used to find trajectories of bifurcating fingers in Sec. V.

\section{LAPLACIAN GROWTH OF NEEDLES AND FINGERS}

\section{A. Loewner's equation}

The Laplacian growth of curved fingers or straight needles is represented by slits growing from the real axis and penetrating the upper half of the $z=x+i y$ plane. Laplace's equation $\Delta \phi=0$ is satisfied in the region exterior to the slits, with boundary condition $\phi=0$ on the slits and real axis. The "hydrodynamic" condition is imposed at infinity: $\phi \rightarrow y$ as $z \rightarrow \infty$. Let $w=g_{t}(z)$ be the map from the slit upper half of the $z$ plane to the entire upper half of the $w$ plane and let $z=f_{t}(w)$ be its inverse (see Fig. 1). The map is normalized by requiring

$$
g_{t}(z)=z+\frac{K(t)}{z}+O\left(\frac{1}{z^{2}}\right) \quad \text { as } \quad z \rightarrow \infty,
$$

where $K(t)$ is a real function of time. In the case of a single slit the map $g_{t}(z)$ satisfies the (chordal) Loewner equation (e.g., [19])

$$
\dot{g}_{t}(z)=\frac{d(t)}{g_{t}(z)-a(t)},
$$

where $a(t)$ is the (real) driving function and $d(t)=\dot{K}(t)$. Note that $a(t)$ is the image of the finger or needle tip in the $w$ plane. The solution for $\phi$ is immediate once the map $g_{t}$ is determined since $\phi=\operatorname{Im}(w)=\operatorname{Im}\left(g_{t}\right)$. It is usual to consider the growth problem such that the finger has zero length at $t=0$ so that $\left.g_{t}\right|_{t=0}:=g_{0}(z)=z$ is the identity map, and $a(0)$ is the starting location of the finger on the real $z$ axis. For $N$ fingers (2) generalizes to (e.g., $[9,19,20])$.

$$
\dot{g}_{t}(z)=\sum_{k=1}^{N} \frac{d_{k}(t)}{g_{t}(z)-a_{k}(t)} .
$$

There is an extensive literature on the chordal Loewner equation (2), and its radial counterpart, including the case when the driving function is stochastic. See, for example, reviews by Gruzberg and Kadanoff [26] and Bauer and Bernard [18]. Here the focus is on the case when the driving function is deterministic, and such that the slits either follow curved paths which ensure the diffusive field is locally symmetric at their tips (geodesic fingers), or when they are straight needles growing in prescribed directions.

\section{B. Growth speed of a finger or needle}

At the tip $z=\gamma$ of the finger or needle, the gradient of $\phi$ has the usual inverse square root singularity:

$$
|\nabla \phi(\gamma+r, t)| \sim \frac{C(t)}{\sqrt{r}},
$$

where $r$ is a local radius from $z=\gamma[14,20]$ and $C(t)$ is a positive function of time. By integrating the flux of $\phi$ into the tip it can be shown $[14,20]$ that the speed of the tip $v$ is given by $v=|C(t)|$. Further, if the map from the upper half of the $w$ plane to the slit upper half $z$ plane is $z=f_{t}(w)$ it can be shown $[8,14,20]$

$$
v=\left|f_{t}^{\prime \prime}(a(t))\right|^{-1 / 2} \text {. }
$$

More generally, the speed of the finger or needle can be expressed as $v=|C(t)|^{\eta}=\left|f^{\prime \prime}(a(t))\right|^{-\eta / 2}$ where $\eta$ is a constant power; see, e.g., [8,20]. The value of $\eta$ influences the dynamics. For example, Derrida and Hakim [14] make the choice $\eta=1$ [referred to here as the "natural" choice since it arises from direct integration of the flux about the tip, i.e., Eq. (4)] in a study of radial needles growing from a common origin. They showed that longer needles grow at the expense of shorter needles. This screening effect is inherent in Laplacian growth and is largely responsible for the 

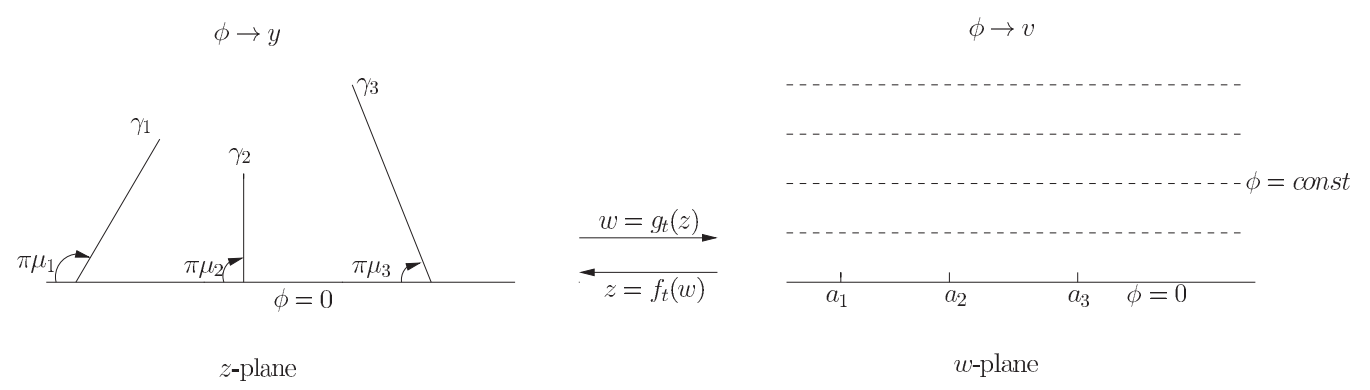

FIG. 1. Mapping between the slit $z$ plane and the upper half of the $w$ plane. The slits in the form of straight needles have tips at $z=\gamma_{i}$ which map to real parameters $w=a_{i}, i=1,2,3$. In the $z$ plane $\phi=0$ on the needles and real $z$ axis, and $\phi \rightarrow y$ as $y \rightarrow \infty$. The needles make angles $\mu_{1} \pi, \mu_{2} \pi$, and $\mu_{3} \pi$ with the real axis. The solution to $\Delta \phi=0$ in the $w$ plane is $\phi=\operatorname{Im}(w)=v$. Contours of $\phi$ in the $w$ plane are dashed lines parallel to the real axis.

typical pattern formation in which long, dominant fingers are observed in a variety of physical scenarios.

Depending on the choice of $\eta$, the screening effect is also present in the growth of multiple fingers governed by Loewner's equation (see [8,20]). In the case of $N$ fingers, the relationship between the Loewner growth rate $d_{k}(t)$ and $v_{k}$ is [20]

$$
\left|d_{k}(t)\right|=\left|f_{t}^{\prime \prime}\left(a_{k}(t)\right)\right|^{-1-\eta / 2} .
$$

For the special case $\eta=-2$, (6) shows $d_{k}(t)$ is constant and no screening occurs as fingers grow with constant speeds. However, for other choices of $\eta$, including the natural choice $\eta=1$, screening results. The effect of $\eta$ on bifurcating fingers is investigated numerically in Sec. V.

\section{NEEDLE GROWTH IN THE HALF PLANE}

\section{A. Numerical method based on the SC Toolbox}

Since the direction of needle growth is prescribed by the initial conditions, just the growth rate of needles is required in order to compute their evolution. Consider $N$ needles growing upward in the $z=x+i y$ plane at arbitrary angles to the real axis. The "natural choice" of screening parameter $\eta=1$ is made, so that each needle grows at a speed according to (5). Figure 1 shows a sketch of two straight needles making angles $\pi \mu_{1}$ and $\pi \mu_{2}$ with the real axis, along with a curved finger (see Sec. IV for discussion of curved fingers) growing in the upper half of the $z$ plane.

The solution of Laplace's equation

$$
\Delta \phi=0,
$$

in the upper half $z$ plane exterior to the needles, such that $\phi=0$ on the needles and real $z$ axis, and satisfying $\phi \rightarrow y$ as $z \rightarrow \infty$ is readily obtained using the inverse SchwarzChristoffel map $w=f_{t}^{-1}(z)=g_{t}(z)$ where the solution is simply $\phi=\operatorname{Im}(w)$. For $N$ needles the Schwarz-Christoffel map is given by

$$
z=f_{t}(w)=A \int_{w_{0}}^{w} \prod_{i=1}^{N} \frac{\left(s-a_{i}\right)}{\left(s-a_{i L}\right)^{\mu_{i}}\left(s-a_{i R}\right)^{1-\mu_{i}}} d s+f\left(w_{0}\right),
$$

where $\pi \mu_{i}$ is the angle the $i$ th needle makes with the negative real axis. The parameters $a_{i L}$ and $a_{i R}$ lie on the real $w$ axis to the left and right of $a_{i}$ respectively, and the ordering $a_{i L}<a_{i}<a_{i R}<a_{(i+1) L}<a_{i+1}<a_{(i+1) R}, i=$ $1, \ldots, N-1$ applies. As the slits grow the parameters $a_{i L}(t), a_{i}(t), a_{i R}(t), i=1, \ldots, N$ vary with time. The real scale factor $A=A(t)$ is also a time-varying map parameter.

In general the Schwarz-Christoffel map of the type (8) contains two arbitrary parameters. Here the choice $A=1$ is made ensuring that the hydrodynamic condition at infinity $\phi \rightarrow y$ as $y \rightarrow \infty$ is satisfied. The other choice made is $a_{1 L}=-a_{N R}$, so that the unknown parameters are located in the interval $\left[-a_{N R}, a_{N R}\right]$ along the real $w$ axis.

By expanding (8) as $w \rightarrow \infty$ the condition that $z \rightarrow w+$ $O\left(w^{-1}\right)$ gives the following relation for the parameters:

$$
\sum_{i=1}^{N} a_{i}-\mu_{i} a_{i L}+\left(1-\mu_{i}\right) a_{i R}=0
$$

Note that the SC Toolbox makes a different choice in assigning the two arbitrary parameters, namely $a_{1 L}=-1$ and $a_{N R}=+1$ and retains $A$ as a parameter. This implies $z \rightarrow A w$ as $w \rightarrow \infty$. In order to satisfy the hydrodynamic condition at infinity computations from the SC Toolbox must therefore be rescaled.

For $N \geqslant 2$ fingers the map parameters $a_{i}, a_{i L}, a_{i R}, i=$ $1, \ldots, N$ are difficult to find explicitly. The SC Toolbox is an efficient way to accurately compute them, provided the spacing between needles is such that numerical inaccuracies owing to "crowding" is not problematic (see Sec. IV D).

The growth speed of the needles (5) requires the second derivatives $f_{t}^{\prime \prime}\left(a_{i}\right), i=1, \ldots, N$ be computed. This is done numerically using the SC Toolbox by computing

$$
f_{t}^{\prime \prime}\left(a_{i}\right)=\lim _{\epsilon \rightarrow 0}\left[f_{t}^{\prime}\left(a_{i}+\epsilon\right)-f_{t}^{\prime}\left(a_{i}-\epsilon\right)\right] / 2 \epsilon,
$$

where $\epsilon>0$ is a small parameter; a typical value used here is $10^{-7}$. Note the first derivatives in (10) are computed straightforwardly and to high accuracy by the SC Toolbox since they are simply the derivatives of the integral form of the map (8), i.e., its integrand. For a small increment in time $\Delta t$ the needle tips are advected in the along-needle direction by the local tip velocity using a time-stepping routine. After each step the map parameters are recomputed enabling the local tip velocities to be updated. Example computations are presented in Sec. III C. 


\section{B. Analytical approach: Two needles}

In the case of needle growth in the half plane it is possible to derive a system of coupled ODEs for the SchwarzChristoffel parameters which when solved determine the needle lengths as functions of time. The ODE system is derived in this section for two needles using ideas from Tsai [22]; see also [27] for the case of $N$ interacting fingers growing parallel to the sides of a semi-infinite strip. The key idea is to model needles as Loewner fingers satisfying (3) and then to find the functional form of driving functions $a_{k}(t), k=1, \ldots, N$ which gives straight fingers.

Consider two needles with lengths $h_{1,2}(t)$ growing upwards from $z= \pm L$ making angles $\pi \mu_{1}$ and $\pi \mu_{2}$ with the real $z$ axis as in Fig. 1. The Schwarz-Christoffel map from the upper half of the $w$ plane to the upper half of the $z$ plane slit by the needles is

$$
f_{t}(w)=\int_{a_{1 L}}^{w} \frac{\left(s-a_{1}\right)\left(s-a_{2}\right)}{\left(s-a_{1 L}\right)^{\mu_{1}}\left(s-a_{1 R}\right)^{1-\mu_{1}}\left(s-a_{2 L}\right)^{\mu_{2}}\left(s-a_{2 R}\right)^{1-\mu_{2}}} d s-L .
$$

Note that the choice $a_{1 L}=-a_{2 R}$ is not explicitly imposed in (11). This simply means the ODE system has an additional firstorder ODE to solve. The corresponding Loewner equation for $g_{t}$ and that satisfied by its inverse $f_{t}$ are, from (3),

$$
\dot{g}_{t}=\frac{d_{1}}{g_{t}-a_{1}}+\frac{d_{2}}{g_{t}-a_{2}}
$$

and

$$
\dot{f_{t}}=-f_{t}^{\prime}\left[\frac{d_{1}}{w-a_{1}}+\frac{d_{2}}{w-a_{2}}\right]
$$

where $f_{t}^{\prime}=d f_{t} / d w$.

Let $I(w)=f_{t}^{\prime}(w)^{2}$ be the square of the integrand in (11):

$$
I=\frac{\left(w-a_{1}\right)^{2}\left(w-a_{2}\right)^{2}}{\left(w-a_{1 L}\right)^{2 \mu_{1}}\left(w-a_{1 R}\right)^{2-2 \mu_{1}}\left(w-a_{2 L}\right)^{2 \mu_{2}}\left(w-a_{2 R}\right)^{2-2 \mu_{2}}} .
$$

Note that $I^{\prime} / I=2 f_{t}^{\prime \prime} / f_{t}^{\prime}$ and, using (13),

$$
\frac{\dot{I}}{I}=\frac{2 f_{t}^{\prime} \dot{f}_{t}^{\prime}}{f_{t}^{\prime 2}}=-\frac{I^{\prime}}{I}\left[\frac{d_{1}}{w-a_{1}}+\frac{d_{2}}{w-a_{2}}\right]+2\left[\frac{d_{1}}{\left(w-a_{1}\right)^{2}}+\frac{d_{2}}{\left(w-a_{2}\right)^{2}}\right]
$$

Direct differentiation of (14) with respect to time gives

$$
\frac{\dot{I}}{I}=-\frac{2 \dot{a}_{1}}{w-a_{1}}-\frac{2 \dot{a}_{1}}{w-a_{2}}+\frac{2 \mu_{1} \dot{a}_{1 L}}{w-a_{1 L}}+\frac{\left(2-2 \mu_{1}\right) \dot{a}_{1 R}}{w-a_{1 R}}+\frac{2 \mu_{2} \dot{a}_{2 L}}{w-a_{2 L}}+\frac{\left(2-2 \mu_{2}\right) \dot{a}_{2 R}}{w-a_{2 R}},
$$

while differentiating (14) with respect to $w$ gives

$$
\frac{I^{\prime}}{I}=\frac{2}{w-a_{1}}+\frac{2}{w-a_{2}}-\frac{2 \mu_{1}}{w-a_{1 L}}-\frac{2-2 \mu_{1}}{w-a_{1 R}}-\frac{2 \mu_{2}}{w-a_{2 L}}-\frac{2-2 \mu_{2}}{w-a_{2 R}} .
$$

Substituting (17) into (15) yields an alternative expression for $\dot{I} / I$ which when equated to (16) and the six limits $w \rightarrow a_{i}, a_{i L}, a_{i R}$, $i=1,2$, considered separately, yields six coupled first-order ordinary differential equations for the Schwarz-Christoffel parameters:

$$
\begin{aligned}
\dot{a}_{j} & =\left(2 \delta_{1 j}-1\right) \frac{d_{1}+d_{2}}{a_{1}-a_{2}}+\frac{\mu_{1} d_{j}}{a_{1 L}-a_{j}}+\frac{\left(1-\mu_{1}\right) d_{j}}{a_{1 R}-a_{j}}+\frac{\mu_{2} d_{j}}{a_{2 L}-a_{j}}+\frac{\left(1-\mu_{2}\right) d_{j}}{a_{2 R}-a_{j}}, \\
\dot{a}_{j L} & =\sum_{i=1}^{2} \frac{d_{i}}{a_{j L}-a_{i}}, \quad \dot{a}_{j R}=\sum_{i=1}^{2} \frac{d_{i}}{a_{j R}-a_{i}},
\end{aligned}
$$

for $j=1,2$.

Recall that the velocity of a needle is given by $\dot{h}_{i}=v_{i}=\left|f^{\prime \prime}\left(a_{i}\right)\right|^{-1 / 2}, i=1,2$, and that (6) implies $d_{i}(t)=\left|f^{\prime \prime}\left(a_{i}\right)\right|^{-3 / 2}$. From (11)

$$
\left|f_{t}^{\prime \prime}\left(a_{i}\right)\right|=\frac{\left|a_{1}-a_{2}\right|}{\left|\left(a_{i}-a_{1 L}\right)^{\mu_{1}}\left(a_{i}-a_{1 R}\right)^{1-\mu_{1}}\left(a_{i}-a_{2 L}\right)^{\mu_{2}}\left(a_{i}-a_{2 R}\right)^{1-\mu_{2}}\right|} .
$$

Thus knowledge of the Schwarz-Christoffel parameters at any given time determines the needle velocities via (19). In practice integrating the needle velocity to find $h_{i}(t)$ is straightforward. Alternatively having computed the SchwarzChristoffel parameters, the integral (11) can be evaluated numerically to find $h_{i}=\operatorname{Im}\left[f_{t}\left(a_{i}\right)\right]$.
The set (18) is straightforward to solve using standard numerical routines for coupled first-order ODEs. Condition (9) serves as a useful check on the accuracy of the solution. Even in this alternative ODE approach there is need, however, to use the SC Toolbox once at the beginning of the procedure to determine the initial values of the Schwarz-Christoffel 
parameters. The initial $z$-plane finger having lengths $h_{1}(0)$ and $h_{2}(0)$ (usually assumed small) are mapped to the upper half of the $w$ plane using the SC Toolbox. The parameters $a_{1}(0), a_{1 L}(0), a_{1 R}(0), a_{2}(0), a_{2 L}(0), a_{2 R}(0)$ are outputs of the map computed by SC Toolbox, taking care, as previously noted, to scale these outputs so that the far-field condition $\phi \rightarrow y$ as $y \rightarrow \infty$ is satisfied.

It is straightforward to extend this approach to $N$ interacting needles; the Appendix gives, for example, the system of ODEs satisfied by the Schwarz-Christoffel parameters for a system of $N$ parallel needles all making right angles to the real $z$ axis.

\section{Examples of two needle growth}

The evolution of two needles can be computed by either (i) the analytical method of Sec. III B involving the solution of system (18) and using this solution to compute the needle velocities $v_{i}=\left|f^{\prime \prime}\left(a_{i}\right)\right|^{-1 / 2}$ and hence lengths at any instant of time, or (ii) employing the SC Toolbox approach described in Sec. III A, where the SC Toolbox is invoked at every time step in order to compute the instantaneous needle velocities. Both methods were tested and found to agree with each other to high accuracy.

Consider two parallel needles of lengths $h_{1}$ and $h_{2}$ growing in the pure imaginary direction from $z= \pm 1$. For small times when the needle lengths are small their effect on each other is negligible and the needles effectively grow independently. For larger times the needles compete with each other and screening occurs. This unstable situation leads to the larger needle growing with the smaller slowing and eventually coming to a halt. These behaviors for two needle pairs are quantified in the following subsections.

\section{Equal length needles}

Let $\mu_{1}=\mu_{2}=1 / 2$ so both needles grow upward from the real axis parallel to the imaginary axis. Letting $h_{1}=h_{2}=h$ and choosing the origin of the $z$ plane to lie equidistant from each needle it follows from (8) that $w_{0}=0$ and $a_{2}=-a_{1}=$ $a,-a_{1 L}=a_{2 R}=a_{R}$, and $-a_{1 R}=a_{2 L}=a_{L}$. The SchwarzChristoffel map (8) then becomes

$$
z=f_{t}(w)=\int_{0}^{w} \frac{\left(s^{2}-a^{2}\right)}{\sqrt{\left(s^{2}-a_{L}^{2}\right)\left(s^{2}-a_{R}^{2}\right)}} d s .
$$

For needles distance two apart (20) gives

$$
\begin{aligned}
i h & =\int_{a_{L}}^{a} \frac{\left(s^{2}-a^{2}\right)}{\sqrt{\left(s^{2}-a_{L}^{2}\right)\left(s^{2}-a_{R}^{2}\right)}} d s \\
& =-\int_{a}^{a_{R}} \frac{\left(s^{2}-a^{2}\right)}{\sqrt{\left(s^{2}-a_{L}^{2}\right)\left(s^{2}-a_{R}^{2}\right)}} d s, \\
1 & =\int_{0}^{a_{L}} \frac{\left(s^{2}-a^{2}\right)}{\sqrt{\left(s^{2}-a_{L}^{2}\right)\left(s^{2}-a_{R}^{2}\right)}} d s .
\end{aligned}
$$

For small time the needle lengths are correspondingly small and hence from (21) $a_{L} \rightarrow a$. Letting $a_{L}=(1-\epsilon) a$, where $\epsilon \ll 1$, it follows from (9) that $a_{R}=(1+\epsilon) a$, and from (21) $a=O(1)$ and $h=a \epsilon+O\left(\epsilon^{2}\right)$. In the same limit (19) gives

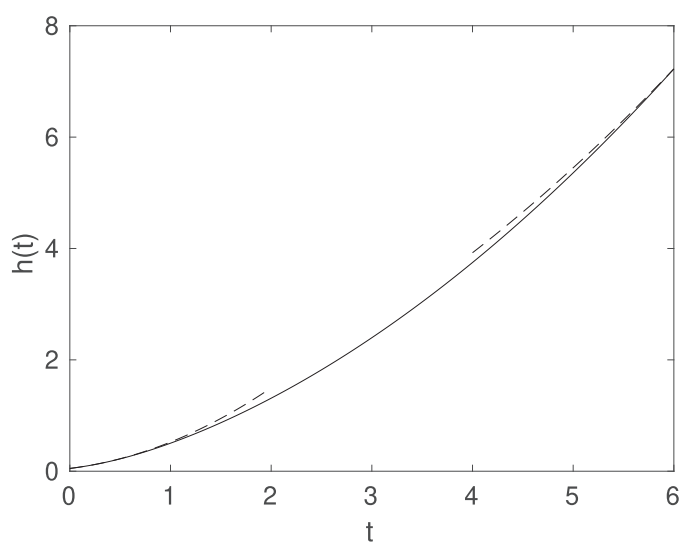

FIG. 2. Growth of two needles of equal length $h_{1}=h_{2}=h(t)$ (solid line). Superimposed are asymptotic length functions for small time, $h \sim t^{2} / 4$, and large time, $h \sim t^{2} / 4 \sqrt{2}+$ const (both dashed lines).

$z^{\prime \prime}(a)=(a \epsilon)^{-1}+O(1)$. Hence using (5), to leading order, $\dot{h}=h^{1 / 2}$ giving $h \sim t^{2} / 4$ growth for small time. Note that this is a different growth rate from the typical $\sqrt{t}$ growth law for an isolated needle for Loewner's equation with constant forcing (6) $d(t)=$ const (see, e.g., [19]) since $\sqrt{t}$ growth results from the choice $\eta=-2$ rather than $\eta=1$ used here.

For large times when $h \gg 1$ (21) implies $a_{L} \ll a \ll a_{R}$ and hence $\pi a^{2}=2 a_{R}$, and $h=a_{R}$ to leading order. The growth speed of the needles is now given by $\dot{h}=\left|f_{t}^{\prime \prime}(a)\right|^{-1 / 2} \approx$ $\left(a_{R} / 2\right)^{1 / 2} \approx \sqrt{h / 2}$, giving $h \sim t^{2} / 4 \sqrt{2}$ growth, a factor of $\sqrt{2}$ slower than the initial growth speed.

Figure 2 shows the length $h_{1}=h_{2}=h(t)$ of the needles as a function of time computed using the SC Toolbox method described in Sec. III A to find the velocity at the tips at any instant and using the Adams-Bashforth time-stepping routine to advance the tips. Also shown is the asymptotic relations for needles with small length $h \sim t^{2} / 4$ and for needles of large length $h \sim t^{2} / 4 \sqrt{2}+$ const, where the constant is chosen so that $h(t)$ and its large length approximation coincide at $t=6$.

\section{Long needles having different lengths}

In this asymptotic analysis it is convenient to rescale (11) using $a_{2 R}>0$ so that $a_{1 L} \rightarrow-1$ and $a_{2 R} \rightarrow+1$. The rescaling reintroduces the scaling parameter $A=a_{2 R}$ and gives the Schwarz-Christoffel map,

$$
z=f_{t}(w)=A \int_{-1}^{w / A} \frac{(s-a)(s-d)}{\sqrt{\left(s^{2}-1\right)(s-b)(s-c)}} d s-1,
$$

where $a=a_{1} / a_{2 R}, b=a_{1 R} / a_{2 R}, c=a_{2 L} / a_{2 R}, d=a_{2} / a_{2 R}$. Hence

$$
\begin{aligned}
i h_{1} & =A \int_{-1}^{a} \frac{(s-a)(s-d)}{\sqrt{\left(s^{2}-1\right)(s-b)(s-c)}} d s \\
& =-A \int_{a}^{b} \frac{(s-a)(s-d)}{\sqrt{\left(s^{2}-1\right)(s-b)(s-c)}} d s, \\
i h_{2} & =A \int_{b}^{c} \frac{(s-a)(s-d)}{\sqrt{\left(s^{2}-1\right)(s-b)(s-c)}} d s \\
& =-A \int_{d}^{1} \frac{(s-a)(s-d)}{\sqrt{\left(s^{2}-1\right)(s-b)(s-c)}} d s .
\end{aligned}
$$




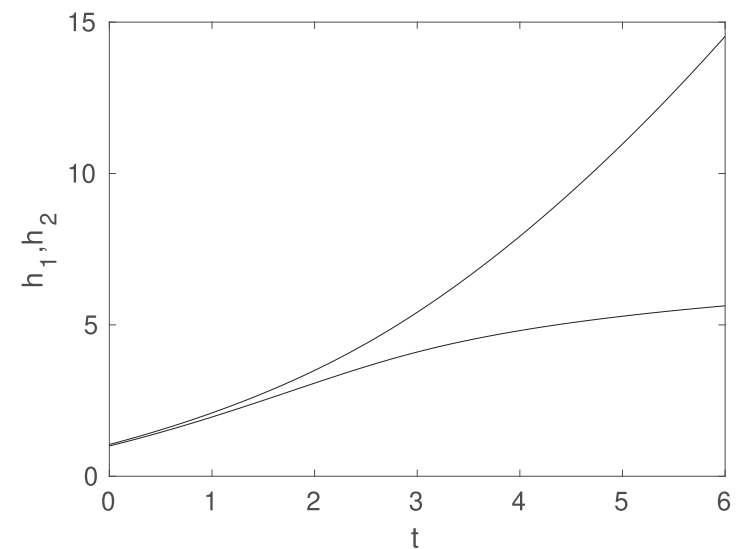

FIG. 3. Lengths of two needles as functions of time with initial lengths 1.0 and 1.05 .

In the two long and equal needle limit considered above $b, c \rightarrow 0$ and $a=-d$. Here the same limit is considered but the parameters are perturbed so that $a \rightarrow a^{*}=-d-$ $\epsilon, b, c \rightarrow b^{*}=c^{*}=-2 \epsilon$, and $d \rightarrow d^{*}=d-\epsilon$, where $0<$ $\epsilon \ll d<1$. The perturbed parameters satisfy the constraint (9). Substituting into (23) gives to leading order in $\epsilon$,

$$
h_{1}-h_{2}=-4 d^{2} A \epsilon \int_{d}^{1} \frac{d s}{s^{2} \sqrt{1-s^{2}}}+O\left(\epsilon^{2}\right) .
$$

Since the right hand side of (24) is negative, the effect of the perturbation is to increase the needle length $h_{2}$ with respect to $h_{1}$. In the same limit the ratio of the square of the speeds of the needles is from (19) to leading order,

$$
\frac{v_{2}^{2}}{v_{1}^{2}}=1+\frac{2 \epsilon}{d\left(1-d^{2}\right)},
$$

and so $v_{2}>v_{1}$. Thus the taller needle grows more quickly than the shorter needle. Consequently the equal length distribution is unstable to small perturbations. This instability, which is evident for needle pairs of any length and not just the long needle limit considered in this analysis, is well known for Laplacian growth, including radial needle growth, e.g., [14]. Figure 3 demonstrates this instability with an example computation of the evolution of $h_{1,2}(t)$ with initial tip locations $-1+1.0 i$ and $1+1.05 i$. The growth rate of the shorter needle approaches zero, while the other grows with ever-increasing speed.

\section{Two inclined needles}

It is straightforward to use the numerical method to quantitatively study the evolution of inclined needles making arbitrary angles with the real axis. While most previous studies of needle growth have considered needles which emanate at right angles to the real axis or needles growing radially outward from a central point, numerical experiments on diffusion limited aggregation suggest that secondary needles may branch at angles other than 90 degrees from primary branches, e.g., [28,29]—-see their Figs. 2 and 3 respectively. Recently Krapivsky et al. [30] considered multiple needle growth in a half space with growing needles making arbitrary angles to a substrate. Such a situation may occur when needles grow

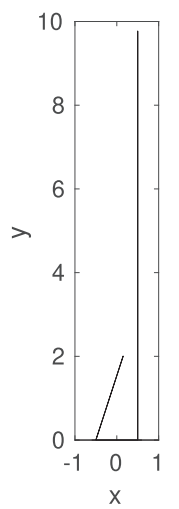

(a)

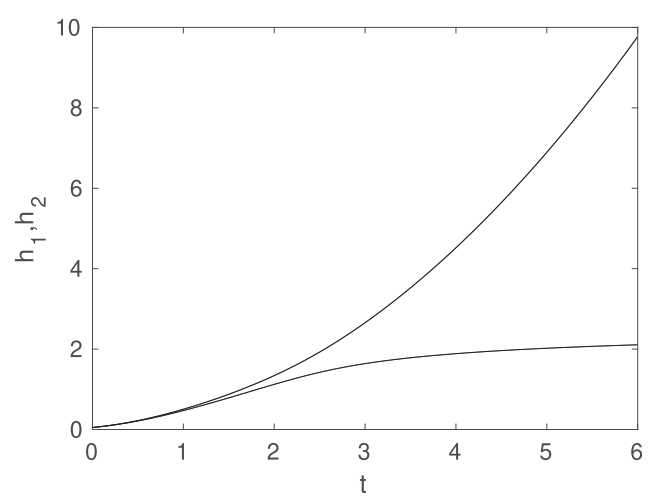

(b)
FIG. 4. (a) Snapshot of a pair of needles at $t=6$. The initial lengths of the needles were both 0.05 . One grows at right angles to the real axis and the other inclined at angle $\mu \pi=3 \pi / 5$. (b) The needle lengths as functions of time.

from a substrate which is not perfectly flat but, rather, has small, random topographic variations. While their paper did not consider Laplacian growth (their needles only interact upon collision), they remark on the application of inclined needle growth to crystal growth.

In this section pairs of inclined needles growing in a half space are considered. Generally, when needles are angled away from each other they continue to grow unimpeded for all time. For needles angled such that they approach each other it is typical for one of the needles to slow allowing the other to pass. The slower needle is then effectively screened and comes to a halt, while the other continues to grow. Figure 4 shows a typical example. Both needles have the same initial length $h_{1,2}=0.05$ and are initially a unit distance apart. The left needle is inclined slightly from the vertical: $\mu_{1}=0.6$, while $\mu_{2}=0.5$. Figure 4(a) shows a snapshot of the needles after $t=6$ and Fig. 4(b) shows their length as a function of

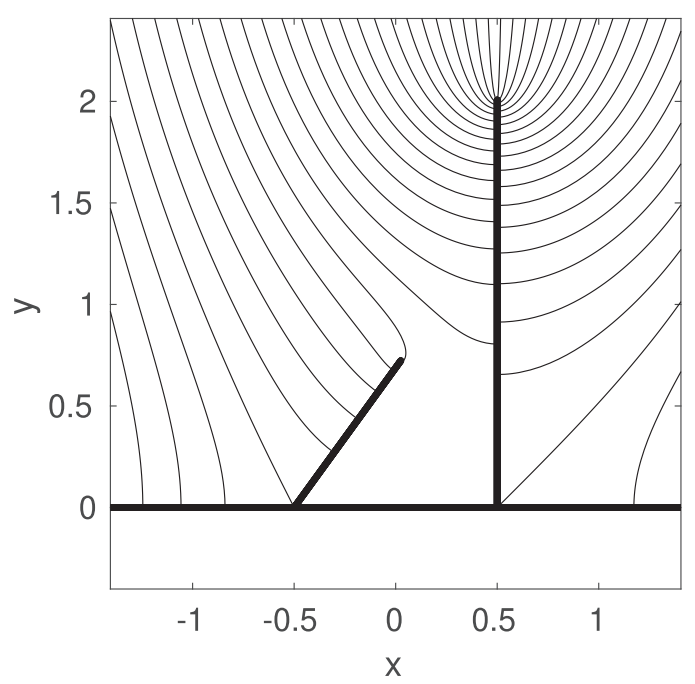

FIG. 5. Contours of the stream-function field at $t=2.5$ for the same two needle experiment shown in Fig. 4. The stream-function values on the contours are uniformly spaced. 


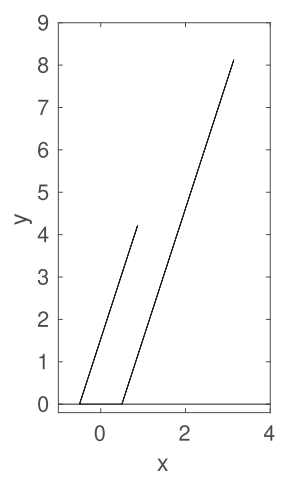

(a)

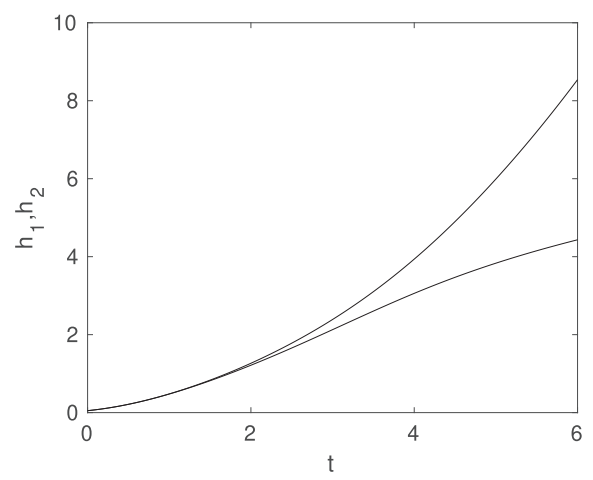

(b)
FIG. 6. (a) Snapshot of a pair of needles at $t=6$. The initial lengths of the needles were both 0.05 ; both grow at angle $\mu \pi=$ $3 \pi / 5$. (b) The needle lengths as functions of time.

time. The screening effect is demonstrated in Fig. 5 which shows uniformly spaced contours of the stream function (the conjugate function of the potential, contours of which are readily obtained using the SC Toolbox) for the same pair of needles at time $t=2.5$. The concentration of stream lines entering the tip of the larger needle in comparison to the shorter needle indicates a greater flux entering the large needle and hence its more rapid growth.

Figure 6 shows two parallel needles with the same angle of incline $\mu_{1,2}=0.6$ and with the same initial lengths $h_{1,2}=$ 0.05 and a unit distance apart. Ultimately screening occurs with one of the needles slowing and the other continuing to grow. As expected, it is the left needle that is screened. This behavior is replicated for other angles of parallel needle pairs starting with the same lengths: if $\mu_{1,2}>0.5$ then the left needle is screened and for $\mu_{1,2}<0.5$ it is the right needle which is screened, i.e., the "inside" needle is the one that ultimately grows the quickest.

\section{E. $N>2$ needles}

Figure 7 shows nine needles each separated by a unit distance growing parallel to the imaginary axis. The needles all start with the same length $h_{i}(0)=0.05, i=1, \ldots, 9$. As expected the needles grow symmetrically about the central needle with the outermost needles growing at the fastest rate, since they are influenced by the other needles on one side only. Since the outermost needles grow quickest it follows that the other needles are eventually screened to a degree proportional to their distance from the outer needles. Hence the resulting pattern illustrated in Fig. 7(a) in which the interior needle tips form a smooth profile with maximum length in the center. Figure 7(b) shows that at about $t=8$ the interior needles have stopped growing while the outermost needles continue to grow. Similar patterns arise for any number of equally spaced needles with the same starting lengths. If the number of needles is even, then the two central needles grow in tandem at the same rate.

The evolution of needles in the semistrip geometry $|x|<$ $1, y>0$ with zero flux boundary condition $\phi_{x}=0$ on $x=$ $\pm 1, y>0$ can also be computed using the method. This is achieved by the same sequence of mappings used by [20]. First, the semi-infinite strip and needles are mapped to the upper half of the $w$ plane by the SC Toolbox. Second, the upper $w$ plane is mapped to the empty semi-infinite strip in the $\zeta$ plane using the map $\zeta=(2 / \pi) \sin ^{-1} w$. The combination of the two maps $z=f_{t}(\zeta)$ is to map the $z$ plane with needles in a semi-infinite strip to an empty semi-infinite strip and the tips $z=\gamma_{i}$ of the needles map to the real $\zeta$ axis, i.e., $\gamma_{i}=f\left(\alpha_{i}\right)$ where $\left|\alpha_{i}\right|<1, i=1, \ldots, N$. The velocity of each tip is given by $\left|f_{t}^{\prime \prime}\left(\alpha_{i}\right)\right|^{-1 / 2}$ which is computed numerically as before.

An example computation is shown in Fig. 8: nine needles evenly spaced in the interval $(-1,+1)$ each having initial length $h_{i}(0)=0.05$ grow vertically upwards. In this case the middle needle continues to grow while the other needles eventually stop growing.

\section{GEODESIC FINGER GROWTH: NUMERICAL PROCEDURE}

\section{A. Numerical method}

In geodesic growth fingers follow curved paths determined by field lines of $\phi(x, y)$. Recent work [11,24] has established the equivalence of geodesic growth and fingers that grow such

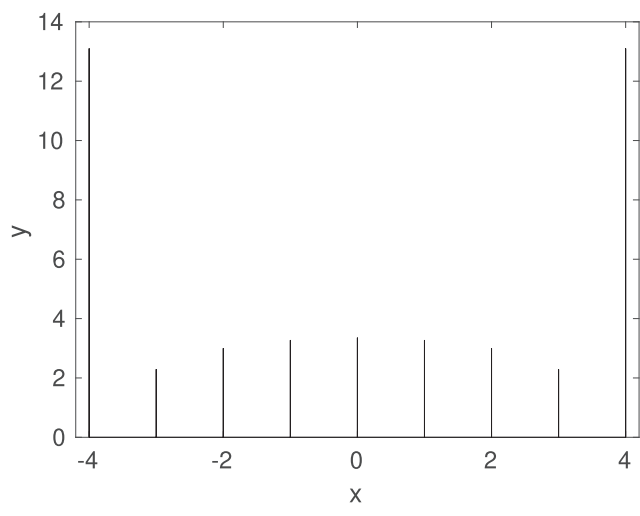

(a)

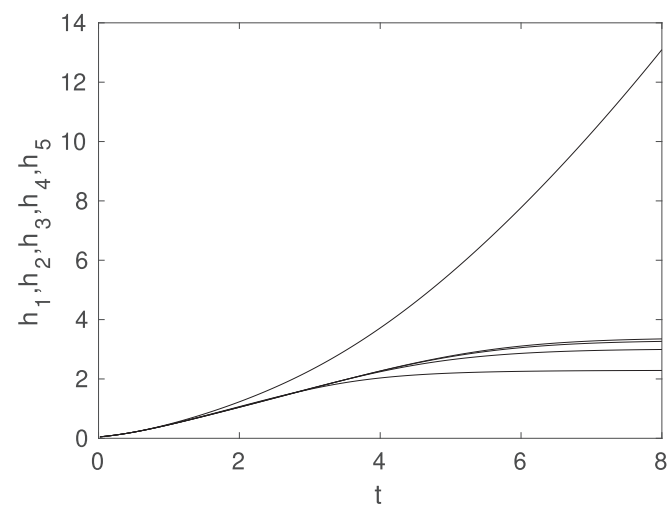

(b)

FIG. 7. (a) Nine evenly spaced needles growing vertically in a half plane. The initial lengths of the needles is 0.05 . The needle lengths maintain a symmetric distribution. (b) Lengths of the needles as function of time. 


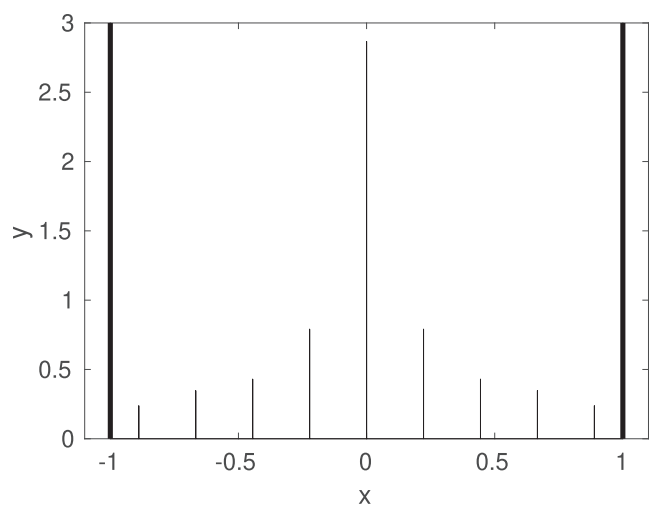

(a)

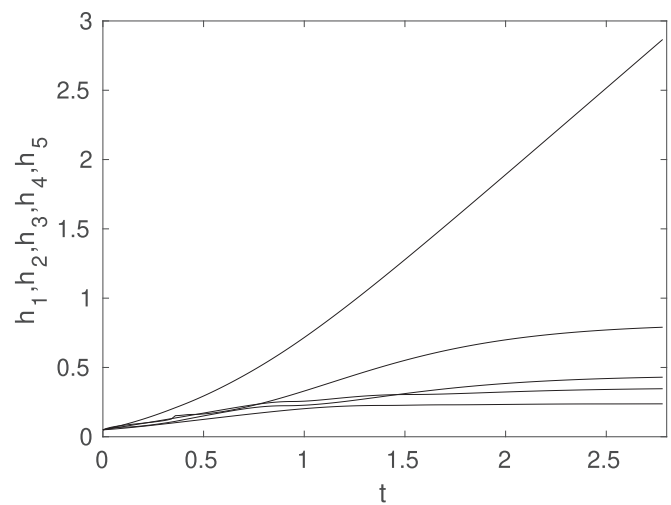

(b)

FIG. 8. (a) Nine evenly spaced needles growing vertically in a semi-infinite strip with zero flux conditions on the vertical sidewalls (shown as thick dark lines). The initial length of the needles is 0.05 . The needle lengths maintain a symmetric distribution. (b) Length of the needles as function of time.

that they maintain local symmetry in the streamlines [i.e., level curves of the conjugate function of the phase $\phi(x, y)]$ entering the tip, and in a direction that maximizes flux into the tip.

By approximating curved fingers as series of small straightline segments — steps — of given length $\delta l$ and treating them as polygons, the SC Toolbox can be employed to model geodesic growth as follows:

(i) At a given time the discretized fingers (which may be growing in a domain with general polygonal geometry, e.g., semistrip, wedge, etc.) are mapped to the upper half of the $w$ plane using the SC Toolbox. As in the needle case, the solution in the $w$ plane is given by $\phi=\operatorname{Im}(w)$, with the $w$-plane image of the $j$ th finger tip $z=\gamma_{j}$ being $a_{j} \in$ Re; see Fig. 9. In the SC Toolbox, $a_{j}$ is computed using the "evalinv" routine.

(ii) The direction at which the next small straight-line increment is oriented is determined by finding the point in the $z$ plane which is given a distance $\delta l$ from the tip, and has the same value of the stream function as the tip $\gamma_{j}$, i.e., the value $a_{j}$. In practice this is done by computing the image $w^{*}$ of points $z^{*}$, where $\left|z^{*}-\gamma_{j}\right|=\delta l$ and such that they lie in a small arc of possible angles on either side of the present direction of the finger (see Fig. 9). The particular point $z^{*}=z^{* *}$ sought is such that the value of its stream function $\operatorname{Re}\left(w^{* *}\right)=a_{j}$ and is found by interpolating between the computed images $w^{*}$. Typically $100 z^{*}$ points are chosen in the arc $[-\pi / 6, \pi / 6]$. If there is only one finger then $z^{* *}$ is the next tip location $\gamma_{j+1}$. For multiple fingers, their relative speeds need to be computed in order to find their relative increment lengths. The procedure is described in the next step.

(iii) The relative growth speeds of multiple fingers are computed using (5) with the second derivative computed according to the limit (10) and the first derivatives calculated using the SC Toolbox "evaldiff" routine. Once the speeds are computed for all tips they are normalized according to $v_{j}=\left|f_{t}^{\prime \prime}\left(a_{j}\right)\right|^{-\eta / 2} / \sum_{k=1}^{N}\left|f_{t}^{\prime \prime}\left(a_{k}\right)\right|^{-\eta / 2}$, to give relative speeds and then each tip is advanced by an increment proportional to their relative speed.

(iv) Once the new straight-line segments have been added to the tips of each finger, the new polygon is mapped to the $w$ plane and the procedure (i)-(iii) is repeated to find the next increment.

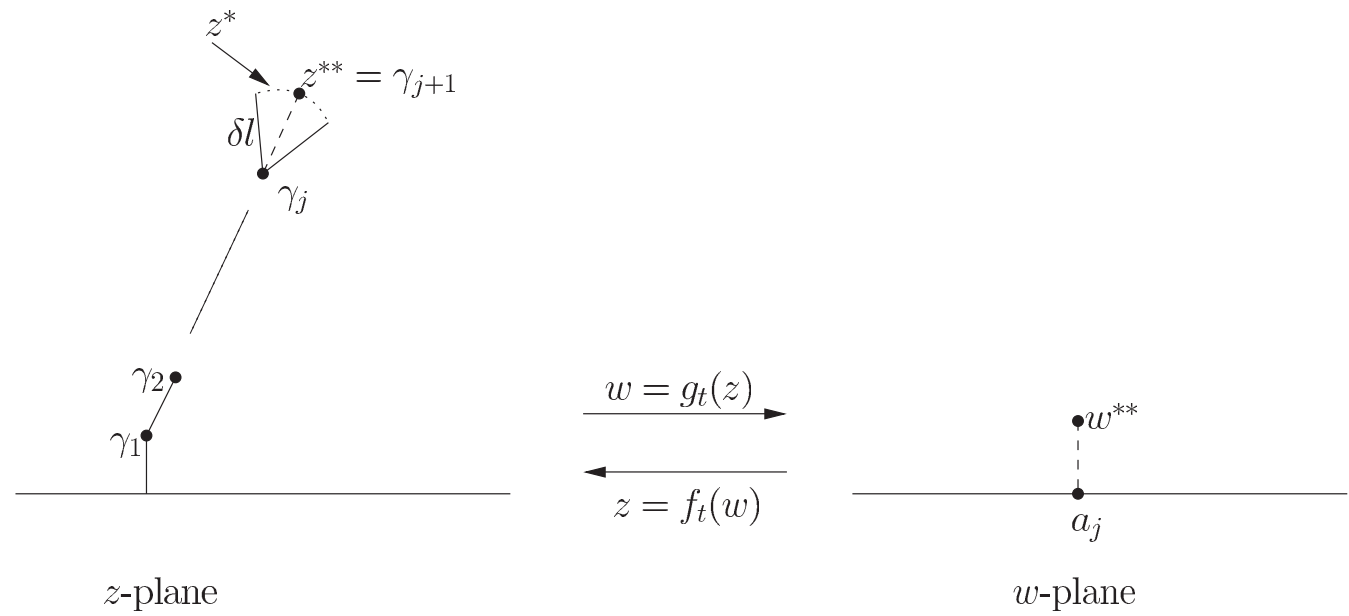

FIG. 9. Mapping between the piecewise straight-line discretized finger in the physical $z$ plane and the upper half of the $w$ plane: $\gamma_{j}$ maps to $a_{j}$ on the $\operatorname{Re}(w)$ axis. The set of points $z^{*}$ lie on an arc of radius $\delta l$ from $\gamma_{j}$. The point $z^{*}=z^{* *}$ is such that $\operatorname{Re}\left(w^{* *}\right)=a_{j}$, where $w^{* *}=g_{t}\left(z^{* *}\right)$. 


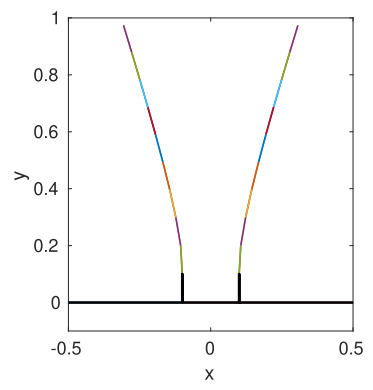

(a)

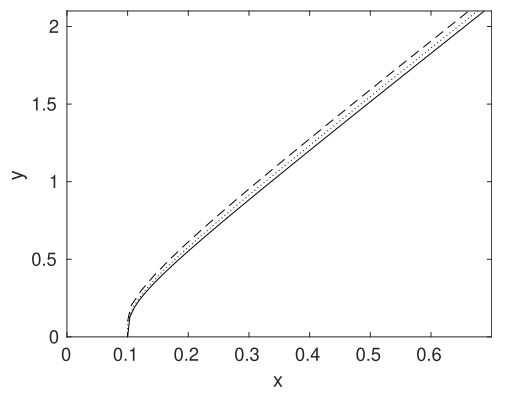

(b)
FIG. 10. (a) Two symmetric fingers growing in a half plane computed using the numerical method. Nine iterations are shown with each step of length 0.1 showing as a different color. The dark line shows the initial starting configuration with fingers 0.2 apart. (b) Shape of the computed right finger for two different step sizes $\delta l=0.05$ (dotted line), 0.1 (dashed line) with 44 and 22 iterations respectively, compared with the exact solution (26) (solid line).

\section{B. Numerical tests}

The numerical procedure is tested by comparing to known exact solutions in two different scenarios: (a) two symmetric fingers growing in a half space [20], and (b) a single finger growing in a semi-infinite strip with zero flux condition on the sidewalls [20]. In (a) the exact solution for the time evolution of the tips $z=\gamma_{ \pm}(t)$ is $[11,20]$

$$
\gamma_{ \pm}\left(\gamma_{ \pm}^{2}-5 a_{0}^{2}\right)^{2}= \pm 16\left(a_{0}^{2}+t\right)^{5 / 2}
$$

where $\gamma_{ \pm}(0)= \pm a_{0}$ correspond to the initial starting points of the fingers on the real $z$ axis. Figure 10(a) shows the computed fingers after nine iterations having evolved from initial steps of length $\delta l=0.1$ perpendicular to the real axis, and spaced $2 a_{0}=0.2$ apart, i.e., $\gamma_{ \pm}(0)=0.1+0.1 i$. A comparison with the exact solution for two different choices of step length $\delta l=0.1,0.05$ is shown in Fig. 10(b), demonstrating that as the step length decreases there is convergence toward the exact solution. Defining the error as the mean of the absolute difference between the numerical solution and the exact solution at the data points (the same error measure as used by [24]), Fig. 11 shows that the error decreases approximately linearly with step length over a range of step lengths. Unlike the needle case where the number of sides of the polygon remains fixed, the finger computation slows in time as the number of elements forming the polygon increases. The SC Toolbox is required to determine an additional three Schwarz-Christoffel parameters per finger at every time step. Eventually the number of elements becomes so large that "crowding" occurs and the method is no longer reliable; see remarks at the end of this section.

The second test problem grows a single geodesic finger in a semistrip of width 2 [20]. The boundary conditions are $\phi=0$ on the finger and $y=0,|x|<1, \phi \rightarrow y$ as $y \rightarrow \infty$ and there is zero flux through the sidewalls so that $\phi_{x}=0$ on $x= \pm 1, y>0$. By mapping the channel to a half space, Gubiec and Szymczak [20] show that the finger satisfies the modified Loewner equation (for suitably scaled time $t$ and irrespective of the choice of screening parameter $\eta$ since there

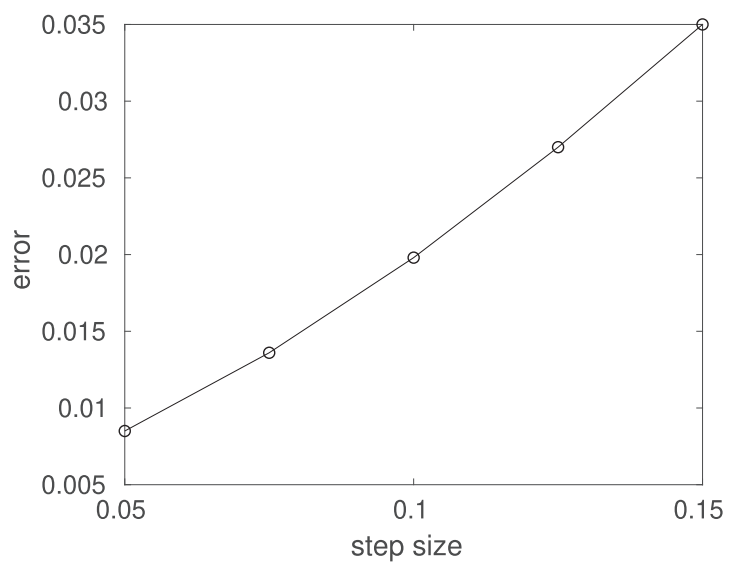

FIG. 11. The average error of the two symmetric finger computation compared to the exact solution as a function of step size $\delta l=0.05,0.075,0.1,0.125,0.15$.

is only one finger)

$$
\dot{g}_{t}=\frac{\pi}{2} \frac{\cos \left(\pi g_{t} / 2\right)}{\sin \left(\pi g_{t} / 2\right)-\sin [\pi a(t) / 2]},
$$

where, for geodesic growth, $a(t)=(2 / \pi) \sin ^{-1}\left[\exp \left(-\pi^{2} t / 8\right)\right.$ $\left.\sin \left(\pi a_{0} / 2\right)\right]$. An implicit solution for the trajectory of the finger can be obtained by solving (27) for $g_{t}$ with the initial condition $g_{0}=z$ and then realizing that the finger tip in the $w$ plane is given by $g_{t}=a$ :

$$
\sqrt{\cos \left(\frac{\pi \gamma}{2}\right)}+\sin \left(\frac{\pi a_{0}}{2}\right) F\left(\frac{\pi \gamma}{4} \mid 2\right)=h(t),
$$

where $F(\sigma \mid m)$ is the incomplete elliptic integral of the first kind and $h(t)$ is an increasing function of time $h(0) \leqslant h(t)<$ $\infty$. The precise form of $h(t)$ is unimportant in determining the shape of the finger trajectory. Note that Gubiec and Szymczak [20] state that such a solution in terms of elliptic function can be found but do not present it.

Figure 12(a) shows the numerically computed finger shape for two different step sizes $\delta l=0.15,0.05$ compared with the analytical solution (28). Figure 12(b) shows the average error as a function of step size. As in the two finger test case, the error decreases approximately linearly with step size. For a step size $\delta l=0.1$, the error is comparable to the high resolution finite-element procedure of Cohen et al. [24] who also performed the same numerical test. Their method also grows the finger by successively adding discrete steps which satisfy local symmetry, the difference being that they directly compute $\phi(x, y)$ in the semistrip using a finite element method.

\section{Three finger evolution}

The numerical method can be used to compute multiple fingers evolving in the half plane for arbitrary values of $\eta$. In this section the evolution of three fingers which maintain symmetry about the middle (vertical) finger is computed for the choices $\eta=-2,1,4$. For $\eta=-2$ finger growth rates are constant and there is an analytic solution for the finger shapes obtained by direct solution of Loewner's equation (3) [21]: denoting the left and right tips by $\gamma_{1}(t)$ and $\gamma_{3}(t)$, the 


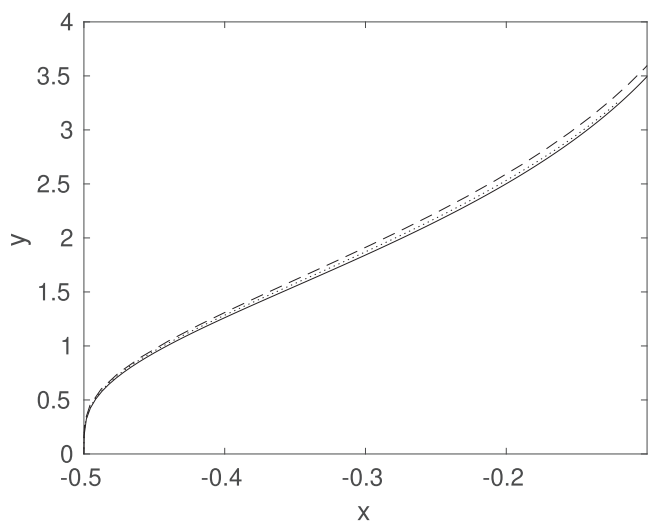

(a)

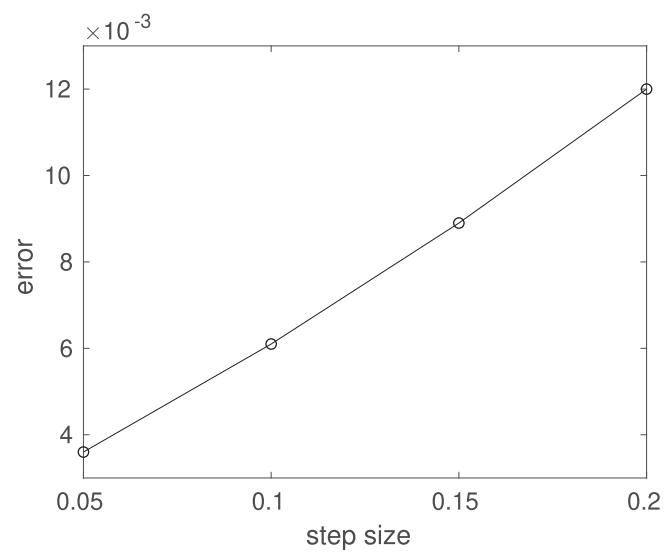

(b)

FIG. 12. (a) Shape of finger starting at $x=-0.5$ and growing in a semi-infinite strip of width 2 . Shown are numerically computed fingers for two different step sizes 0.15 (dashed line) and 0.5 (dotted line) and the exact solution given by (28) (solid line). (b) The average error compared to the exact solution as a function of step size.

trajectories are solutions of the algebraic equation

$$
\begin{aligned}
& {\left[\gamma_{1,3}(t)^{2}-a_{0}^{2} \beta_{+}\right]^{1+\alpha}\left[\gamma_{1,3}(t)^{2}-a_{0}^{2} \beta_{-}\right]^{1-\alpha}} \\
& \quad=\left(1-\beta_{+}\right)^{1+\alpha}\left(1-\beta_{-}\right)^{1-\alpha}\left(a_{0}^{2}+3 d_{0} t\right)^{2},
\end{aligned}
$$

where $\alpha=\sqrt{3 / 19}, \beta_{ \pm}=(9 \pm \sqrt{57}) / 6, d_{0}$ is the constant growth rate, and $\pm a_{0}$ are the starting locations of the finger tips $\gamma_{1,3}$. Note that (29) corrects a minor typographic error in Eq. (14) of [21], namely the presence of the $a_{0}^{2}$ terms inside the square brackets on the left hand side. For the choice $\eta=-2$ the numerical results compare well (not shown) to the exact solution (29). For $\eta \neq-2$ the finger growth rates are not constant, screening may occur, and there are no exact solutions.

Figure 13 superimposes trajectories of the fingers for three different numerical experiments: $\eta=-2,1,4$, with $a_{0}=1$. As in the needle case, screening is evident for $\eta=1$ with growth of the middle needle suppressed in comparison to the outer needles (cf. Fig. 7). Given the middle needle has less influence on the outer fingers, they pursue more vertical paths in comparison to the $\eta=-2$ case. The screening effect is even more pronounced for $\eta=4$, with a relatively small middle needle and more vertical outer needles.

\section{Remarks on numerical crowding}

The numerical method based on the SC Toolbox provides an accurate and efficient computational approach since it accounts for the singularity at the finger tip by mapping the whole finger to the real axis of the $w$ plane. Its main limitation is the number of steps that can be added before crowding prevents accurate numerical calculation of the SchwarzChristoffel map - that is, when the parameters of the SchwarzChristoffel map on the real $w$ axis become exponentially close (see user guide for the SC Toolbox [25]). For example, in the test example of this section involving semi-infinite strip geometry crowding becomes problematic when the number of steps with $\delta l=0.1$ reaches 55 . In contrast, for the same step size, in the symmetric two finger evolution in the half-plane experiment, the number of elements in each finger can be grown well beyond 80. It is likely that the reason for this is that the fingers in this case grow away from each other. No crowding is evident in the results reported in Sec. III on needle growth over the time scales shown, although for larger times crowding is evident especially when fingers are very long compared to the separation distance of their tips. In general, the numerical problem of crowding depends on the geometry and shape of the fingers. The present numerical method is not well suited for application to, say, whole stream networks with many interacting streams (which involves a large number of Schwarz-Christoffel parameters). It is, however, useful in

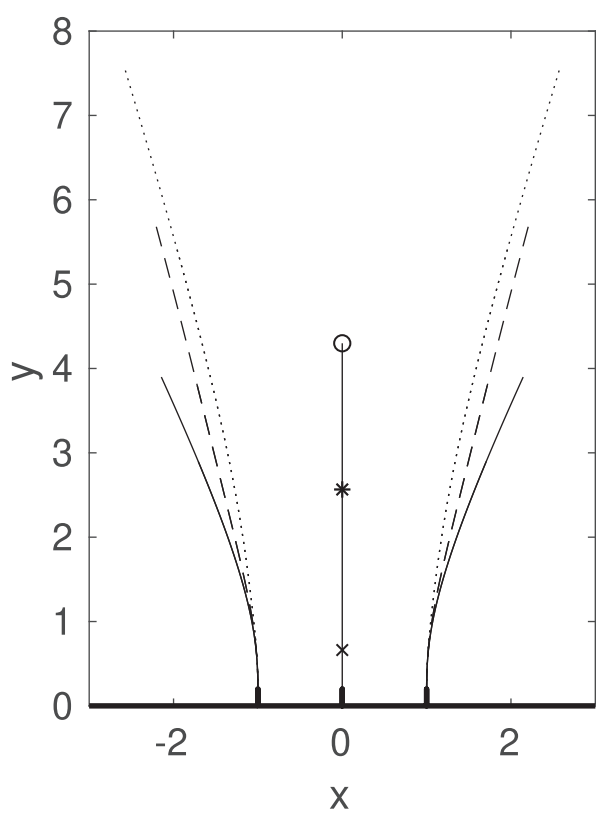

FIG. 13. Snapshots of the trajectories taken by three fingers which are symmetric about the middle finger. Three different numerical experiments are shown corresponding to $\eta=-2$ (solid line), 1 (dashed line), 4 (dotted line). The tip of the middle finger is denoted in each case by $\eta=-2(\circ) ; \eta=1(*) ; \eta=4(\times)$. The small vertical solid black ticks on the real axis represent the initial heights of the fingers. 


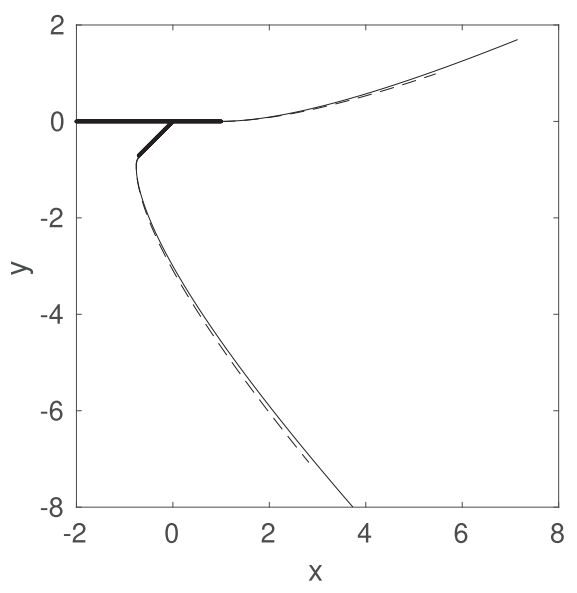

(a)

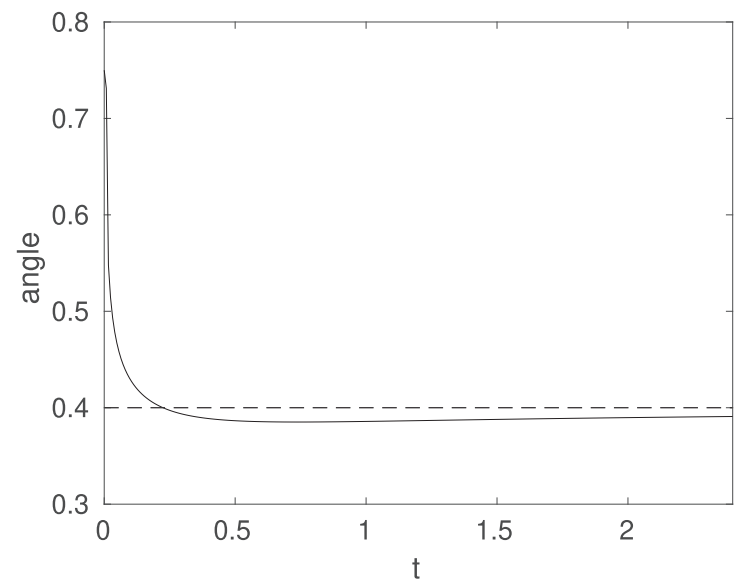

(b)

FIG. 14. (a) Comparison of exact finger trajectories (solid line) obtained by conformal mapping of (26) for $t=2.4$, and those obtained by the numerical method (dashed lines). The initial secondary bifurcation and the primary semi-infinite needle are the thick solid lines. (b) The angle between the tangents of the finger tips as a function of time. The dashed line is the $2 \pi / 5$ angle.

understanding local phenomena such as interaction of fingers with boundaries and when they bifurcate. The latter application is considered in the next section.

\section{BIFURCATING FINGERS}

The remarkable observation that groundwater-fed streams tend to bifurcate at an angle of $2 \pi / 5$ has attracted much attention, e.g., $[3,10,11]$. Groundwater flow in these networks is approximated by the solution of Laplace's equation $\Delta \phi=0$ subject to $\phi=0$ on the streams and with uniform flux at infinity. Assuming streams to be infinitesimally thin, the use of Loewner's equation to model their growth is appropriate.

Carleson and Makarov [8] showed that for two needles of finite length ("secondary" needles) branching symmetrically from a semi-infinite needle (the "primary" needle) the bifurcation angle $2 \pi / 5$ is special since it is the unique angle for which the secondary needles are geodesic, i.e., $\phi$ is locally symmetric about the tips (see also [3,9-11,31]). More generally, the solution (26) describing a pair of fingers growing symmetrically in the half plane can be mapped to two secondary fingers bifurcating from a primary semi-infinite needle: Devauchelle et al. [11] showed, for arbitrary initial symmetric bifurcating angles, the secondary fingers asymptote toward a $2 \pi / 5$ bifurcation angle, and that this angle is a stable fixed point.

For fingers bifurcating asymmetrically from a semi-infinite needle the same mapping approach can be used provided the fingers grow at the same constant rate. To do this first, the "exact" finger trajectories are found for a nonsymmetric initial angle of bifurcation using the same procedure as used in [11]: suppose the primary needle lies along the negative real axis and bifurcates at the origin with one finger extending unit distance along the positive real axis and a second branch extending unit distance along the ray $r \exp (-3 \pi / 4)$; see Fig. 14(a). Note that there is no length scale in this problem so the initial lengths of the perturbations are taken to be of a unit length. The initial bifurcation (the thick lines of Fig. 14) is mapped to the real axis of the $w$ plane using the
SC Toolbox, with the tips of the secondary fingers mapped to $w= \pm a_{0}$. The solution (26) is then the solution in the $w$ plane and is mapped back (using the map computed by the SC Toolbox) to giving the solid line trajectories in Fig. 14. Care needs to be taken to ensure the correct scaling is made when using the SC Toolbox, since in the semi-infinite needle case the far-field condition $w \rightarrow z^{1 / 2}$ for large $z$ applies, so the scaling of the computed Schwarz-Christoffel parameters involves $\sqrt{A}$ instead of $A$ as in the half-plane case. This is essentially the same procedure as [11] except that here the SC Toolbox is used to compute the map since its analytic form is not explicitly known in the asymmetric case.

Comparison is made in Fig. 14(a) between trajectories found by the conformal mapping procedure and those computed numerically by growing the fingers with screening parameter $\eta=-2$, so that from (6) $d_{1,2}=$ const. The step size is $\delta l_{1}+\delta l_{2}=\delta l=0.2$. The agreement between the two methods is reasonable. Also plotted in Fig. 14(b) is the angle between the fingers as a function of time, as measured by the tangential directions of the fingers at their tips. As expected this angle approaches $2 \pi / 5$.

When $\eta \neq-2$ growing fingers must be computed numerically since the mapping procedure does not apply since it relies on the symmetric case where fingers grow at the same rate. Figure 15 considers an initial $\pi / 2$ bifurcation from a semi-infinite primary needle and shows the evolution of secondary fingers for three different numerical simulations with $\eta=-2,1,4$. Clearly the trajectories are dependent on choice of $\eta$ : with $\eta=-2$ (solid line) the growth rate of each finger is the same and the fingers evolve toward a bifurcation angle of $2 \pi / 5$. For the natural choice of $\eta=1$ one of the fingers is noticeably longer with the finger initially at right angles to the primary needle being screened to some extent. In the $\eta=-4$ case there is very strong screening and the finger from the right-angled branch starting at $z=-i$ is so short it does not appear in the figure. As a result there is only a small deviation of the other finger as it continues largely in the same direction as the primary finger, and the bifurcation angle stays close to $\pi / 2$. 


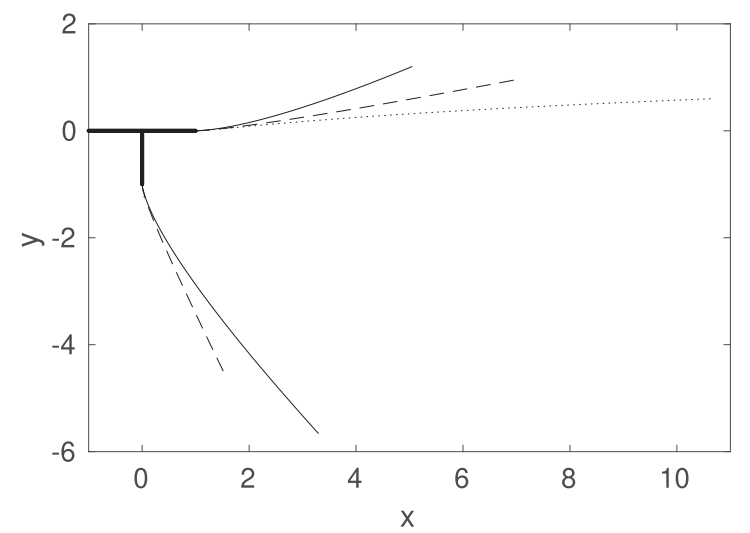

FIG. 15. Screening of fingers from an initial right-angled bifurcation (thick solid line) from a semi-infinite needle for $\eta=-2$ (solid line), 1 (dashed line), 4 (dotted line). Shown are the shape of the fingers in each case after 100 iterations. In the case $\eta=4$ the finger growing from the right-angle starting at $z=-i$ is barely noticeable.

\section{CONCLUSION}

This paper presents effective analytical and numerical methods for studying Laplacian growth of fingers and needles in the upper half plane. The methods share the use of the Schwarz-Christoffel map, this being the "natural" map to use in the case of needles which form polygonal regions. Curved fingers are approximated as polygons by discretization.

Knowledge of the conformal map from the empty upper $w$ plane to the upper $z$ plane slit by needles enables the growth speed of the needles to be expressed explicitly in terms of the second derivative of the map. The asymptotic behavior of pairs of needles is deduced from the form of the Schwarz-Christoffel map. When the screening parameter $\eta=$ 1 , the results demonstrate the fundamental Laplacian growth instability, namely that longer needles grow at the expense of shorter needles. Screening also occurs when needles propagate toward each other or when they propagate parallel to each other but are inclined to the real $z$ axis. The SC Toolbox provides an accurate way of computing the map and hence the growth of the needles. The numerical method is able to compute $N \geqslant 2$ interacting needles either in the upper half plane or in a semistrip. Alternatively, following [22,27], a set of ODEs governing the parameters of the Schwarz-Christoffel map is derived whose solution for needle growth agrees with the numerical method.

By discretizing fingers into straight-line segments their growth and interaction is similarly considered. Fingers which bifurcate from a semi-infinite needle are of interest in the ramification of stream networks and are considered here as an example of the numerical method. It is shown that asymmetric bifurcations experience screening and that the finger trajectories depend on the screening parameter $\eta$.

The numerical method for growing fingers and needles is straightforward to adapt to other geometries provided they are polygonal, e.g., the Laplacian growth of fingers in wedges and growing from finite polygons can be readily computed. For growth governed by partial differential equations (PDEs) other than Laplace's equation the method may still be use- ful. Although such PDEs are likely not to be conformally invariant, the fact that the Schwarz-Christoffel transformation accurately accounts for the singularity at the tips is a powerful feature of the present method. The drawback is that after mapping a nontrivial PDE needs to be solved in the $w$ plane which although in a simple domain is likely to need solving by other numerical methods, e.g., spectral methods. Even so, this combined approach has been successfully used in other problems, e.g., the computation of point vortex motion near sharp corners governed by the modified Helmholtz equation [32].

Another extension of this work would be to use the methodology to consider "fat slits" [33,34] in which Loewnertype evolution is applied to fingers and slits which are not vanishingly thin. For example, Durán and Vasconcelos [34] consider the growth of fat fingers in a channel, a scenario which mimics interfacial fingering instability in a channel.

\section{ACKNOWLEDGMENTS}

C. Millar helped develop the numerical method based on the SC Toolbox. The useful suggestions made by the reviewers are gratefully acknowledged.

\section{APPENDIX: $N$ PARALLEL NEEDLES}

For $N$ needles growing parallel to the imaginary axis in the upper half of the $z$ plane the map (8) has time-dependent Schwarz-Christoffel parameters: $a_{k}, a_{k L}, a_{k R}, k=1, \ldots, N$ where $a_{k}$ is the preimage in the $w$ plane of the tip of the $k$ th needle. Using a similar method as Sec. III B (see also [27] for the semi-infinite strip geometry case) it can be shown that the parameters satisfy

$$
\begin{gathered}
\dot{a}_{k}=\sum_{j(\neq k)=1}^{N} \frac{d_{k}+d_{j}}{a_{k}-a_{j}}-\frac{d_{k}}{2} \sum_{j=1}^{N}\left(\frac{1}{a_{k}-a_{j L}}+\frac{1}{a_{k}-a_{j R}}\right), \\
\dot{a}_{k L}=\sum_{j=1}^{N} \frac{d_{j}}{a_{k L}-a_{j}}, \quad \dot{a}_{k R}=\sum_{j=1}^{N} \frac{d_{j}}{a_{k R}-a_{j}},
\end{gathered}
$$

for $k=1, \ldots, N$. The $d_{k}$ are given by (6). Also,

$$
2 \sum_{j=1}^{N} a_{j}=\sum_{j=1}^{N}\left(a_{j L}+a_{j R}\right)
$$

which serves as useful check on the solution of system (A1). The needle lengths as a function of time can be deduced from the Schwarz-Christoffel map (8) or by direct integration of the velocities

$$
\dot{h}_{k}=\left|\frac{\prod_{j \neq k}^{N}\left(a_{k}-a_{j}\right)}{\sqrt{\prod_{j=1}^{N}\left(a_{k}-a_{j L}\right)\left(a_{k}-a_{j R}\right)}}\right|^{\eta} .
$$

The initial conditions for $a_{k}, a_{k L}, a_{k R}, k=1, \ldots, N$ are found using the SC Toolbox: at $t=0$ the initial needle lengths $h_{k}(0)$ are known and the resultant polygon is mapped to the upper half of the $w$ plane and the SC Toolbox computes the parameters $a_{k}(0), a_{k L}(0), a_{k R}(0)$. As noted in Sec. III A, care must be taken to rescale the results from the SC Toolbox to ensure that $g_{t} \rightarrow z$ as $z \rightarrow \infty$. 
[1] P. Saffman and G. Taylor, The penetration of a fluid into a porous medium or Hele-Shaw cell containing a more viscous liquid, Proc. R. Soc. London, Ser. A 245, 312 (1958).

[2] T. A. Witten and L. M. Sander, Diffusion-Limited Aggregation, a Kinetic Critical Phenomenon, Phys. Rev. Lett. 47, 1400 (1981).

[3] O. Devauchelle, A. P. Petroff, H. F. Seybold, and D. H. Rothman, Ramification of stream networks, Proc. Natl. Acad. Sci. USA 109, 20832 (2012).

[4] W.-T. Ke, J. B. Shaw, R. C. Mahon, and C. A. Cathcart, Distributary channel networks as moving boundaries: Causes and morphodynamic effects, J. Geophys. Res.: Earth Surf. 124, 1878 (2019).

[5] O. Zik, Z. Olami, and E. Moses, Fingering Instability in Combustion, Phys. Rev. Lett. 81, 3868 (1998).

[6] T. H. Johansen, M. Baziljevich, D. V. Shantsev, P. E. Goa, Y. M. Galperin, W. N. Kang, H. J. Kim, E. M. Choi, M. S. Kim, and S. I. Lee, Dendritic magnetic instability in superconducting $\mathrm{MgB}_{2}$ films, Europhys. Lett. 59, 599 (2002).

[7] C. Giverso, M. Verani, and P. Ciarletta, Branching instability in expanding bacterial colonies, J. R. Soc. Interface 12, 20141290 (2015).

[8] L. Carleson and N. Makarov, Laplacian path models, J. Anal. Math. 87, 103 (2002).

[9] G. Selander, Two deterministic growth models related to diffusion-limted aggregation, Ph.D. thesis, Royal Institute of Technology, Stockholm, 1999.

[10] A. P. Petroff, O. Devauchelle, H. Seybold, and D. H. Rothman, Bifurcation dynamics of natural drainage networks, Philos. Trans. R. Soc., A 371, 20120365 (2013).

[11] O. Devauchelle, P. Szymczak, M. Pecelerowicz, Y. Cohen, H. J. Seybold, and D. H. Rothman, Laplacian networks: Growth, local symmetry, and shape optimization, Phys. Rev. E 95, 033113 (2017).

[12] M. A. Peterson, Nonuniqueness in Singular Viscous Fingering, Phys. Rev. Lett. 62, 284 (1989).

[13] M. A. Peterson and J. Ferry, Spontaneous symmetry breaking in needle crystal growth, Phys. Rev. A 39, 2740 (1989).

[14] B. Derrida and V. Hakim, Needle models of Laplacian growth, Phys. Rev. A 45, 8759 (1992).

[15] Y. Huang, G. Ouillon, H. Saleur, and D. Sornette, Spontaneous generation of discrete scale invariance in growth models, Phys. Rev. E 55, 6433 (1997).

[16] M.-O. Bernard, J. Garnier, and J.-F. Gouyet, Laplacian growth of parallel needles: A Fokker-Planck equation approach, Phys. Rev. E 64, 041401 (2001).
[17] M. Abate, F. Bracci, M. D. Contreras, and S. Daz-Madrigal, The evolution of Loewner's differential equations, Eur. Math. Soc. Newsletter 78, 31 (2010).

[18] M. Bauer and D. Bernard, 2D growth processes: SLE and Loewner chains, Phys. Rep. 432, 115 (2006).

[19] W. Kager, B. Nienhuis, and L. P. Kadanoff, Exact solutions for Loewner evolutions, J. Stat. Phys. 115, 805 (2004).

[20] T. Gubiec and P. Szymczak, Fingered growth in channel geometry: A Loewner-equation approach, Phys. Rev. E 77, 041602 (2008).

[21] M. A. Durán and G. L. Vasconcelos, Interface growth in two dimensions: A Loewner-equation approach, Phys. Rev. E 82, 031601 (2010).

[22] J. Tsai, The Loewner driving function of trajectory arcs of quadratic differentials, J. Math. Anal. Appl. 360, 561 (2009).

[23] T. Kennedy, Numerical computations for the SchrammLoewner evolution, J. Stat. Phys. 137, 839 (2009).

[24] Y. Cohen, O. Devauchelle, H. F. Seybold, R. S. Yi, P. Szymczak, and D. H. Rothman, Path selection in the growth of rivers, Proc. Natl. Acad. Sci. USA 112, 14132 (2015).

[25] T. A. Driscoll, The Schwarz-Christoffel Toolbox for MATLAB, version 2.3, http://www.math.udel.edu/ driscoll/SC/.

[26] I. A. Gruzberg and L. P. Kadanoff, The Loewner equation: Maps and shapes, J. Stat. Phys. 114, 1183 (2004).

[27] M. Pecelerowicz, A. Budek, and P. Szymczak, Effective description of the interaction between anisotropic viscous fingers, Europhys. Lett. 108, 14001 (2014).

[28] J.-P. Eckmann, P. Meakin, I. Procaccia, and R. Zeitak, Growth and form of noise-reduced diffusion-limited aggregation, Phys. Rev. A 39, 3185 (1989).

[29] B. Derrida, V. Hakim, and J. Vannimenus, Growth histories and overlap distributions of diffusion-limited-aggregation clusters, Phys. Rev. A 43, 888 (1991).

[30] P. Krapivsky, L. Nazarov, and M. Tamm, Geometrical selection in growing needles, J. Stat. Mech.: Theory Exp. (2019) 073206.

[31] M. B. Hastings, Growth exponents with 3.99 walkers, Phys. Rev. E 64, 046104 (2001).

[32] O. R. Southwick, E. R. Johnson, and N. R. McDonald, A point vortex model for the formation of ocean eddies by flow separation, Phys. Fluids 27, 016604 (2015).

[33] A. Zabrodin, Growth of fat slits and dispersionless KP hierarchy, J. Phys. A: Math. Theor. 42, 085206 (2009).

[34] M. A. Durán and G. L. Vasconcelos, Fingering in a channel and tripolar Loewner evolutions, Phys. Rev. E 84, 051602 (2011). 